high ambient humidity, was brought to Piracicaba, São Paulo, where it was examined. Kept in the laboratory, in an ambient with a relative humidity level varying from 50 to $70 \%$, the psocids disappeared after a period of 30 to 45 days and the Suidasia pontifica population was much reduced. This observation for the mite species is consistent with its Astigmatid nature, its water balance being essentially dependent on the relative humidity of the environment (or the atmosphere inside the package).

\section{SUMMARY}

Two insect and two mite species are reported from dried and salted fish ("pirarucu", Arapaima gigas Cuvier) in Manaus, Amazonas, Brazil, namely: Liposcelis bostrychophilus Badonnel (Insecta, Psocidae) and the parasite of its eggs, Alaptus globosicornis Girault (Insecta, Hymenoptera, Mymaridae); and, Suidasia pontofica Oudemans (Acari, Astigmata, Saproglyphidae) and Blattisocius keegani Fox (Acari, Mesostigmata, Ascidae). Key words: dried fish, stored-products insects and mites.

\section{REFERENCES}

CICILYKUTTY, A.K.; BALASUBRAMANIAN, N.K. \& JOHN, P.A.

1981a - The influence of physical conditions (salt content) of the flesh of fish on the intensity of infestation by adult mite Suidasia medanensis. Acarologia, 22 (3): 291-293.

$1981 \mathrm{~b}$ - The influence of physical conditions (Moisture content) of the flesh of fish on the intensity of infestation by the adult mite Suidasia medanensis. Acarologia, 22 (3): 295-297.

FAINT, A. \& PHILIPS, J.R.

1978 - Notes on the genus Suidasia Oudemans, 1905 with descriptions of a new species from Australia (Acari, Astigmata, Saproglyphidae).

FLECHTMANN, C.H.W.

1968 - Notas sobre ácaros de produtos armazenados. Solo, Centro Acad. "Luiz de Oliveira", Piracicaba, SP, 60 (1): 63-65.

HUGHES, A.M.

1976 - The Mites of Stored Food and Houses. Min. Agric. Fish. Food, London, Techn. Bul.I, 9. 400pp.

(Aceito para publicação em 19/05/82)

\title{
Presença de alcalóides ß-carbolínicos no Rami ou Hami (Bebida de índio)
}

\section{Wilson Wolter Filho ('), José Augusto da Silva Cabral (') e Arnaldo Imbiriba da Rocha ${ }^{(2)}$}

\section{INTRODUÇÃO}

O homem, mesmo em sociedades mais antigas, sempre fez uso de substâncias naturais com ações alucinogênicas. Ulm grupo considerável destas substâncias, entre elas a harmina e a harmalina apresenta em comum o núcleo indólico, derivado do triptofano. Sua atividade sobre o sistema nervoso é devida à interferência nas funções de determinadas substâncias endógenas, como por exemplo, a serotonina (Wcoley, 1962), que são básicas nas funções bioquímicas do cérebro.

\section{MATERIAL E MÉTodos}

A amostra estudada $(750 \mathrm{ml})$ foi fornecida pelo indigenista da Fundação Nacional do índic (FUNAI), Sebastião Amâncio da Costa, que a adquiriu na tribo dos índios Canamari, habitantes das cabeceiras dos rios Javari, Juruá e afluentes nos Estados do Amazonas e Acre.

Duas espécies vegetais, mantidas em se gredo pelos índios, entram em sua composição, sendo que outras espécies são adicionadas somente com a intençäo de confundir os curiosos. A bebida é difundida entre a maioria das

(1) - Instituto Nacional de Pesquisas da Amazônia, Manaus.

(2) - Fundação Universidade do Amazonas, Manaus. 
nações indígenas da Amazônia Ocidental e seu uso também é adotado por brancos. Via de regrá, é preparada por decocção e utilizada em comemorações festivas e religiosas. Normalmente, é ingerida ao anoitecer e, inicialmente, provoca náuseas e vòmitos. Seu efeito varia de acordo com a concentraçâo dos vegetais portadores de princípios ativos, sendo em média cie 12 horas. Durante este período, ocorrem alucinações e um estado de semiconsciência caracterizado por euforia e incapacidade de controle de movimentos, à semelhança da embriaguez alcoólica. Passado o efeito da bebida, advém um estado de torpor que impossibilita a realização de qualquer atividade, inclu. sive a sexual.

C materia! fornecido foi testado para verificar-se a presença de alcalóides em sua composição, usando-se reagentes de Dragendorff modificado segundo Murnier (Randerath, 1964), apresentando resultado positivo. Em seguida, foi acidificado com ácido tartárico $(\mathrm{pH} \sim 1)$ e extraído exaustivamente com éter sulfúrico. A fração etérea foi concentrada e reservada para estudos posteriores.

A fase aquosa ácida foi alcalinizada com hidróxicio de amônio e extraída (refluxo) com: éter etílico (fração A) e, após, com acetato de etila (fração B.).

As frações orgânicas foram concentradas separadamente e submetidas a novo teste indicando a presença de alcalóides. Foi estudada apenas a fração $A$, por ter apresentado um maior rendimento (em torno cie $90 \%$ ) e ser idêntica às outras, fato constatado por cromatografia em camada delgada de sílica. Desta fração, foram isoladas duas substâncias em placa preparativa de sílica-gel $60 \mathrm{Hf}_{254}$ (Art. 7739, Merck) e como fase móvel clorofórmio/ metanol $(85: 15)$. A primeira $(40 \mathrm{mg})$, cristalina incolor, P.F. $258,5-260^{\circ} \mathrm{C}(\mathrm{MeOH})$, quando exposta à luz uitravioleta mostra fluorescência azul, enquanto que a segunda (15 mg), nas mesmas condições apresenta fluorescência verde. As duas substâncias foram denominadas respectivamente $\mathrm{H}-1$ e $\mathrm{H}-2$.

O composto $\mathrm{H}-1$, foi analisado através de um sistema GC/MS/DS, Finnigan, mod. 4021, acoplado com computador tipo INCOS utilizan- do coluna capilar de silica $(30 \mathrm{~m} \times 0,25 \mathrm{~mm}$, SE-54, WCOT, cujo espectro de massa mostra um pico base em m/z $212(100 \%)$ que corresponde ao ín molecular, além de outros fragmentos como: m/z $197(27 \%), 183(8 \%)$ e $169(66 \%)$.

$\mathrm{H}-1$ foi identificado como sendo 1-metil-7metoxi- $\beta$-carbolina (Harmina) por comparação direta do espectro obtido com o já existente na biblioteca do computador para esta substância.

A estrutura da harmina foi confirmada através da comparação do seu ponto de fusão com o da lit., $261^{\circ} \mathrm{C}$ (dec).: (Paradies, 1975) juntamente com os seguintes dados espectrais de RMNH $\left(60 \mathrm{MHz}, \mathrm{F}_{3} \mathrm{CCOOH}\right.$ ) $)$ e I. V. (KBr) obtidos dessa substância. -RMN'H $(10,12 \delta$, s, $\mathrm{N}-\mathrm{H}),(8,20 \delta, \mathrm{m}, 3 \mathrm{H}),(7,25 \delta, \mathrm{m}, 2 \mathrm{H}),(4,15$ $\left.\delta, \mathrm{s},-\mathrm{O}-\mathrm{CH}_{3}\right)$ e $\left(3,20 \delta, \mathrm{s},-\mathrm{CH}_{3}\right)$. -I.V., $\left(3.340,1610\right.$ e $\left.830 \mathrm{~cm}^{-1}\right)$.

$\mathrm{O}$ espectro de massa de $\mathrm{H}-2$ registrou $\mathrm{O}$ pico molecular em m/z $214(100 \%)$, além de outros fragmentos como m/z 213 (99\%), 199 $(17 \%), 198(23 \%)$ e $170(20 \%)$. As características do espectro no infravermelho mostraram-se similares a $\mathrm{H}-1$, entretanto, sob radiação de luz ultravioleta (366 nm) apresenta fluorescência esverdeada.

Comparando essas informações com os da Literatura (Rivier \& Pilet 1971 e Coutts, et al., 1970 J, sugerimos para H-2 uma estrutura igual ou semelhante à da harmalina. Além disso, o fato de haver registrada a coocorrência de harmina e harmalina em diversas espécies de Banisteriopsis (B. argentea Spring ex Juss., B. caapi (Spruce ex Griseb.) Morton e B. inebrians Morton), assim como em Passiflora incarnata (Passıfloracea), (Alien \& Holmsteat, 1980), poderá reforçar essa suposição.

\section{AgRADECIMENTOS}

Expressamos nossos agradecimentos a Maria do Perpétuo Socorro Sarrafe dos Santos e a Francisca Socorro Nascimento Taveira, bolsistas áo $\mathrm{CNPq} / \mathrm{INPA}$ e à Fundação Nacional do f́ndio (FUNAI) pelo material fornecido. 


\section{SUMMARY}

From a drink widely utilized by the Indian nations of western Amazonia, hallucinogenic, two $\beta$-carbo'inic alkaloids have been isolated.

\section{REFERENNCIAS BIBLIOGRÁFICAS}

ALLEN, J.R.F. \& HOLMSTEAT, B.R.

1980 - The Simple B.Carboline Alcaloids. Phytochem., 19: 1575-7.

COUTTS, R.T.; LOLOCK, R.A. \& SLYWKA, G.W.A.

1970 - Mass Spectra of Selected $\beta$-Carbolines [ $\beta-9 H-P y r i d o ~(3,4-b)$ indoles] Org. Mass Spectrometry, 9: 879-89.
PARADIES, H.

1957 - (Apud) J. Am. Chem. Soc. 79: 5735. In: The Merck Index. 9. ed. U.S.A., Merck \& CO. Inc., 1976. p. 4471.

RANDERATH, $\mathrm{K}$.

1964 - Chromatografie Sur Couches Minces. Paris, Gauthier - Villars.

RIVIER, L. \& PILET, P.

1971 - Composés Hallucinogènes Indoliques. Ann. Biol., 10 (3-4): 138.

WOOLEY, D.H.

1962 - The Biochemical Bases of Psychoses. New York, John Wiley.

(Aceito para publicação em $24 / 05 / 82$ ) 